\title{
EXPLOITING INDEPENDENCE MEASURES IN DUAL SPACES WITH APPLICATION TO ATRIAL F-WAVE EXTRACTION IN THE ECG
}

\author{
R. Phlypo* ${ }^{\star \dagger}$, V. Zarzoso ${ }^{\dagger}$, I. Lemahieu* \\ * IBBT-MEDISIP, Ghent University, Campus Heymans, 185 De Pintelaan, 9000 Ghent, Belgium. ronald.phlypo@ugent.be, \\ ignace. lemahieu@ugent.be, ${ }^{\dagger}$ Lab. I3S, UNSA-CNRS, Les Algorithmes, bât Euclide B, 2000 Route des Lucioles, BP121, \\ 06903 Sophia Antipolis CEDEX, France. zarzoso@i3s.unice.fr
}

Keywords: Independent Component Analysis, Domains, Blind Source Separation, Electrocardiogram

\begin{abstract}
Atrial flutter is a life-threatening cardiac arrhythmia with an increasing prevalence during the last decennia. To characterise and diagnose atrial fibrillation based on the electrocardiographic waveforms, we propose an algorithm that extracts the f-wave from the observations. This algorithm is based on classical independent component analysis algorithms, but is applied in a transform domain of the original signal to assure that the source extraction contrast is discriminative for the source of interest. Results show that it is of high importance to choose the right domain before processing the signal.
\end{abstract}

\section{Introduction}

Atrial Fibrillation (AF) is the most common cardiac arrhythmia encountered in clinical practice. The prevalence of the arrhythmia increases with age reaching as high as $9 \%$ in persons aged 80 years and older [1]. Amongst others, due to the aging population and more frequent monitoring the hospitalisation increased with about $66 \%$. More alarming is the associated augmented rate of mortality with respect to patients in normal sinus rhythm. However, the actions of mechanism are still subject to controversy [2] and more information needs to be gathered about the how and when of the electrical and chemical cardiac activity.

The electrocardiogram (ECG), a visualisation of the electrical activity on the thorax, is a useful non-invasive and inexpensive tool to detect abnormal cardiac rhythms. Since atrial fibrillation is defined as "a supraventricular tachyarrhythmia characterized by uncoordinated atrial activity " [1], the segments in the ECG usually displaying the atrial repolarization (P-wave) and the equipotential line between two consecutive heart beats, are replaced by rapid oscillations or fibrillatory waves (f-waves), see Figure 1. The only condition here is that the atrioventricular junction is functioning regularly. As can be seen from Figure 1, the atrial activity can only be observed over disjoint T-Q time intervals, making it difficult to have sufficiently specific frequency information (cf. the Gabor uncertainty principle). Moreover, during the QRS-T interval, the atrial activity is present, but masked by the much larger in amplitude ventricular activity. The presented method will be based on the ergodicity of the signal statistics as to recover the masked activity and to separate - as a grateful side-effect - the f-waves from background ECG noise.

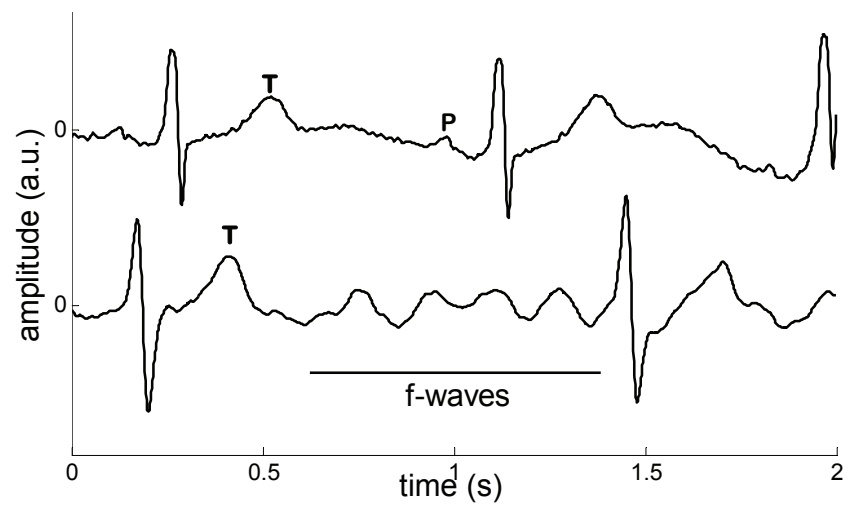

Figure 1: Example of Normal Sinus Rhythm (upper) and Atrial Fibrillatory wave (lower)

The model used in this paper is the linear instantaneous mixing model, wherein temporal observations $\boldsymbol{y}(t) \in \mathbb{C}^{n}$ can be given as the result of a linear mixing of $m \leq n$ sources $s_{i}(t) \in \mathbb{C}, i=1 \ldots m$. The linear mixing channels are given as $\boldsymbol{A}=\left[\begin{array}{lll}\boldsymbol{a}_{1} & \ldots & \boldsymbol{a}_{m}\end{array}\right], \boldsymbol{a}_{i} \in \mathbb{R}^{n}$, or in matrix presentation

$$
\boldsymbol{y}(t)=\boldsymbol{A s}(t)
$$

Our aim is to estimate the flutter source (without loss of generality we can identify the source as $\boldsymbol{x}_{1}$ ) and the corresponding mixing channel $\boldsymbol{a}_{1}$ by inversing the system:

$$
\boldsymbol{x}(t)=\boldsymbol{B}^{-1} \boldsymbol{y}(t)=\boldsymbol{B}^{-1} \boldsymbol{A} \boldsymbol{s}(t) .
$$

Since the ventricular and atrial activities have no instantaneous statistical dependence, these signals could be estimated by a class of algorithms known as Independent Component Analysis (ICA) [3]. However, it is well known that this class of algorithms works well for non Gaussian signals being more discriminative when the signal statistics are far from Gaussian. We inform the reader that here and in 
the rest of this paper non Gaussianity and (close to) Gaussianity are used solely with respect to the statistical characteristics of the signals and not necessarily associated with a class of distributions. Moreover, ICA contrasts are generally unable to recover the scale, neither the permutation of the sources, which leads us to select the following ICA contrast $[4,5]$ among the available alternatives:

$$
\Psi_{1}(\boldsymbol{x}(t))=-\kappa_{1}^{\mathrm{x}}+\sum_{\mathrm{i}=1}^{\mathrm{n}} \kappa_{\mathrm{i}}^{\mathrm{x}},
$$

where $\kappa_{i}$ stands for the fourth order marginal cumulant of the $i$-th variable $\boldsymbol{x}_{\boldsymbol{i}}$ (nevertheless, without modification the contrast can be generalised to any arbitrary order $(>2)$ if the cumulant is finite and discriminative on the set $\boldsymbol{x}$ ). It has been shown that this function is a contrast when $\kappa_{1}^{S}$ is negative and all $\kappa_{i \neq 1}^{s}>0$. For the extraction of f-waves, this contrast is suboptimal. This is due to the close to gaussianity statistical properties, i.e. the quasi vanishing cumulants of any order greater than two, of quasi periodic waveforms [3]. As a result, our signal of interest cannot be separated from a background with cumulants that are lower in value than the cumulant of the f-wave. It is not guaranteed that the f-waves have indeed the lowest cumulant value among the sources.

In this paper we propose to transform the signal into a dual domain so as to have a representation where the source of interest has either a limited support - which is negligible with respect to the total support of the observed signal either has a statistical distribution for which the cumulants under consideration are highly discriminative. The domain to choose is closely related to the signal under observation. In this paper we opted for the frequency domain. This choice is not unique but is based on the property of linearity of the transform, which eases the subsequent calculations. The representation of our source of interest in this domain is now supposed to have increased super-gaussianity because the fwaves are quasi periodic, and thus the domain is suitable to be subjected to an analysis for independent sources under an extraction contrast.

\section{Methods}

\subsection{Redefining the contrast and the statistics}

Since we move from the real (time) domain to a complex representation domain, we need to redefine the cumulants as used in the objective function. The Fourier transform, being a linear transform, will not change our (real) mixing matrix $\boldsymbol{A}$ as defined in Eq.(1). But transforming into the Fourier domain changes the choice of the contrast function for source extraction into:

$$
\boldsymbol{\Psi}_{2}(\widetilde{\boldsymbol{x}}(\omega))=\kappa_{1}^{\mathrm{c}}-\sum_{\mathrm{i}=1}^{\mathrm{n}} \kappa_{\mathrm{i}}^{\mathrm{c}},
$$

where the superscript $\kappa_{j}$ denotes the marginal cumulant of $x_{j}$, $\mathcal{C}_{j j j j}^{x}$, in the complex domain. The general fourth order cumulants of a variable $\boldsymbol{x}$ being defined as below:

$$
\begin{aligned}
\mathcal{C}_{\boldsymbol{i} \boldsymbol{j} k \boldsymbol{l} l}^{\boldsymbol{x}} \stackrel{\text { def }}{=} \mathbb{E}\left\{\boldsymbol{x}_{\boldsymbol{i}} \boldsymbol{x}_{\boldsymbol{j}}^{*} \boldsymbol{x}_{\boldsymbol{k}} \boldsymbol{x}_{\boldsymbol{l}}^{*}\right\}-\mathbb{E}\left\{\boldsymbol{x}_{\boldsymbol{i}} \boldsymbol{x}_{\boldsymbol{j}}^{*}\right\} \mathbb{E}\left\{\boldsymbol{x}_{\boldsymbol{k}} \boldsymbol{x}_{\boldsymbol{l}}^{*}\right\} \\
-\mathbb{E}\left\{\boldsymbol{x}_{\boldsymbol{i}} \boldsymbol{x}_{\boldsymbol{k}}\right\} \mathbb{E}\left\{\boldsymbol{x}_{\boldsymbol{j}}^{*} \boldsymbol{x}_{\boldsymbol{l}}^{*}\right\}-\mathbb{E}\left\{\boldsymbol{x}_{\boldsymbol{i}} \boldsymbol{x}_{\boldsymbol{l}}^{*}\right\} \mathbb{E}\left\{\boldsymbol{x}_{\boldsymbol{j}}^{*} \boldsymbol{x}_{\boldsymbol{k}}\right\} .
\end{aligned}
$$

In the above equation, $\mathbb{E}\{$.$\} is the mathematical expectation$ operator, and the subscripted star denotes the complex conjugate. Consider now all cumulants of a signal pair $\left(x_{p}, x_{q}\right)$. These cumulants are equal under a permutation $\pi(i, k)$ and/or $\pi(j, l)$ in the above definition, and are complex conjugate under the permutations $\pi(i, j), \pi(i, l), \pi(j, k)$ and/or $\pi(k, l)$, which will prove useful in the next section.

\subsection{Optimising the contrast $\Psi_{2}$}

It has been empirically shown that a contrast in $m$ variables can be optimised using consecutive updates over all possible variable pairs, also known as Jacobi updates using Givens rotations [6]. Consider $\boldsymbol{z}=\boldsymbol{H}^{\mathbf{- 1}} \boldsymbol{y}$, where $\boldsymbol{H}$ is any matrix that whitens the variables $\boldsymbol{y}$, i.e. $\boldsymbol{z}$ is decorrelated and each entry has unit variance, or $\mathbb{E}\left\{\mathbf{z Z}^{\boldsymbol{H}}\right\}=\boldsymbol{I}$. I stands for the identity matrix in $\mathbb{R}^{m \times m}$ and ${ }^{H}$ denotes the transposed conjugate or Hermitian. The Fourier transform being linear we have

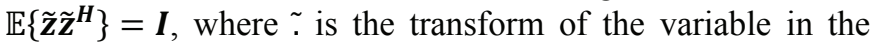
frequency domain. Remains us to find a unitary transform, connecting the separator output $\boldsymbol{x}$ to $\boldsymbol{z}$, say $\boldsymbol{x}=\boldsymbol{Q}^{T} \boldsymbol{z}$ or, equivalently, $\widetilde{\boldsymbol{x}}=\boldsymbol{Q}^{T} \tilde{\boldsymbol{z}}$. The multilinearity of the cumulants now permits us to connect the cumulants of the separator output to those of the input as

$$
\kappa_{j}^{c}=\sum_{p, q, r, s=1}^{m} \boldsymbol{Q}_{p j} \boldsymbol{Q}_{q j} \boldsymbol{Q}_{r j} \boldsymbol{Q}_{s j} \mathcal{C}_{p q r s}^{z}
$$

Recall that we only process pairs of signals [6] and thus we can reduce the above to

$$
\kappa_{j}^{c}=\sum_{k=1}^{4}\left(\begin{array}{l}
4 \\
k
\end{array}\right) \boldsymbol{Q}_{p j}^{4-k} \boldsymbol{Q}_{q j}^{k} \overline{\mathcal{C}}_{k(4-k)}^{\left(z_{p} z_{q}\right)},
$$

where the "cumulants" are defined as

$$
\begin{gathered}
\overline{\mathcal{C}}_{40}^{\left(z_{p} z_{q}\right)}=\mathcal{C}_{p p p p}^{\left(z_{p} z_{q}\right)} \\
\overline{\mathcal{C}}_{31}^{\left(z_{p} z_{q}\right)}=\Re\left(\mathcal{C}_{p p p q}^{\left(z_{p} z_{q}\right)}\right)
\end{gathered}
$$

$$
\begin{gathered}
\overline{\mathcal{C}}_{22}^{\left(z_{p} z_{q}\right)}=\frac{\Re\left(\mathcal{C}_{q p q p}^{\left(z_{p} z_{q}\right)}\right)+2 \mathcal{C}_{p p q q}^{\left(z_{p} z_{q}\right)}}{3} \\
\overline{\mathcal{C}}_{13}^{\left(z_{p} z_{q}\right)}=\Re\left(\mathcal{C}_{p q q q}^{\left(z_{p} z_{q}\right)}\right) \\
\overline{\mathcal{C}}_{04}^{\left(z_{p} z_{q}\right)}=\mathcal{C}_{q q q q}^{\left(z_{p} z_{q}\right)}
\end{gathered}
$$

and where the symbol $\mathfrak{R}$ stands for the real part of its argument. With this definitions, any ordinary ICA algorithm considering pairwise cumulants can be employed to optimise the contrast function in the frequency domain. As argumented above, we will use the contrast $\boldsymbol{\Psi}_{1}$.

\subsection{Application to the f-waves in atrial flutter ECG}

The flutter waves are characterised by a repeating pattern at a typical rate of $4 \mathrm{~s}^{-1}$ to $5.3 \mathrm{~s}^{-1}$, with a range of $3.3 \mathrm{~s}^{-1}$ to $9 \mathrm{~s}^{-1}$. Based upon this observation and the fact that the flutter rate is 
slowly varying in time, we aim at extracting a source that is highly leptokurtic, i.e. has a high positive kurtic value, in the frequency interval $3-9 \mathrm{~Hz}$. In other words, we use only a part of the total spectrum and more specifically the interval on which we can state with almost certainty that the contrast $\boldsymbol{\Psi}_{2}$ will be discriminative for the source of interest. Our model reduces to

$$
\widetilde{\boldsymbol{x}}(\omega)=\boldsymbol{Q}^{T}\left[\boldsymbol{H}^{-1} \boldsymbol{y}\right](\omega)=\boldsymbol{Q}^{T} \widetilde{\boldsymbol{z}}(\omega), \quad \forall \omega \in \Omega
$$

We denote $\tilde{\boldsymbol{z}}(\omega)=\left[\boldsymbol{H}^{-1} \widetilde{\boldsymbol{y}}\right](\omega)$ with which we want to make clear that the prewhitening matrix $\boldsymbol{H}$ has been calculated on all realisations of $\boldsymbol{y}$ or, equivalently, on its complete spectrum, although any part of the spectrum, $\Omega$, can be used to calculate the rotation matrix $\boldsymbol{Q}$. The algorithm is intuitively equivalent to an optimisation of the spectral concentration around a frequency in the set $\Omega$. A major advantage is that the modal frequency does not need to be known a priori. This can be seen as follows:

Take the definition of spectral concentration

$$
S C=\frac{\int_{0.87 \omega_{m}}^{1.12 \omega_{m}} x_{1}(\omega) x_{1}^{*}(\omega) \mathrm{d} \omega}{\int_{0}^{1 / 2} x_{1}(\omega) x_{1}^{*}(\omega) \mathrm{d} \omega}
$$

$\omega_{m}$ represents the modal frequency in the $3-9 \mathrm{~Hz}$ band. Since we know that the signals are unit variance and are transformed through a unitary transformation, the separator output will be of unit variance and thus the denominator equals 1 . To augment the term in the nominator, all energy should be concentrated into a narrow band (or in several narrow bands if the frequency hops over time). By using ICA on the spectral coefficients, we assure with the chosen contrast that the separator output $x_{1}$ will have a maximally "peaky" distribution, or a minimal support, in the $\Omega$-band. This proofs intuitively the equivalence between a high spectral concentration and a maximally kurtic distribution.

One might wonder whether the spectral concentration, which is obviously not taking the higher harmonics into consideration, can be used to separate the f-waves from the QRS-T waveforms. Under the assumption that the f-waves are indeed the unique/major contributor to the modal frequency $\omega_{m}$, it can be seen that the spatial filter $\boldsymbol{b}_{1}$ that is optimised under the above conditions will be the filter associated to the (approximate) inverse of the mixing vector $\boldsymbol{a}_{1}$ and thus $x_{1} \approx s_{1}$. Since $s_{1}$ contains all harmonics, so will $x_{1}$.

\section{Results}

At first we show how certain waveforms behave in the temporal and frequency domain. Table 1 contains cumulant values for 4 different zero-mean waveforms of unit variance in both domains, together with their spectral concentration. Where appropriate the cumulant is given as a function of the samples in the corresponding domain, i.e. $N$ temporal samples, or $2 N$ Fourier coefficients. The spectral concentration for a triangular wave is a function of the
Riemann zeta function $\zeta($.$) as 100 / \zeta(2)=600 / \pi^{2} \approx$ $60.8 \%$. The spectral cumulant for a triangular wave depends on the order of expansion in Fourier coefficients. It has been empirically observed that for a signal with about 5 harmonics the cumulant is of the order $N / 2$.

\begin{tabular}{|c|c|c|c|}
\hline$f(t)$ & $\kappa(t)$ & $\kappa^{c}(\omega)$ & $S C[\%]$ \\
\hline sinusoid & $-3 / 2$ & $\mathcal{O}(N)$ & 100 \\
triangular & $-6 / 5$ & $\mathcal{O}(N / 2)$ & $600 / \pi^{2}$ \\
Dirac $\delta\left(t_{0}\right)$ & $\mathcal{O}(N)$ & -2 & n. a. \\
$\sim \mathcal{N}(0,1)$ & 0 & -1 & n.a. \\
\hline
\end{tabular}

Table 1: Cumulant values and spectral concentration for analytical signals (n.a. $=$ non-applicable)

Next, we show the algorithm's performance on a synthetic dataset, where we consider the unitary mixture of a periodic wave, a highly supergaussian wave and white non-gaussian noise, as displayed in Figure 2. The resulting observations after unitary mixture are shown in Figure 3. The results are obtained running 100 Monte Carlo realisations of the dataset with an associated unitary mixing matrix. Recall that the unitary mixing matrix permits us to restrict the search to the rotation matrix $\boldsymbol{Q}$, but this is without loss of generalisation to $\boldsymbol{B}$ if the source signals $\boldsymbol{S}$ are perfectly decorrelated.

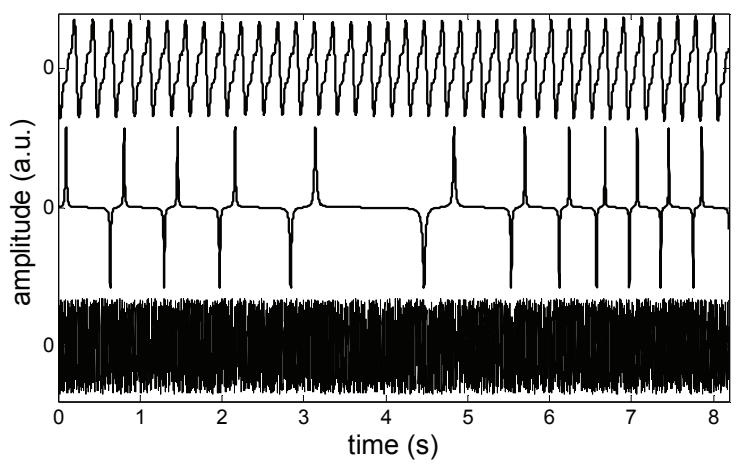

Figure 2: A set of waves, $\boldsymbol{s}$, as used in the synthetic dataset.

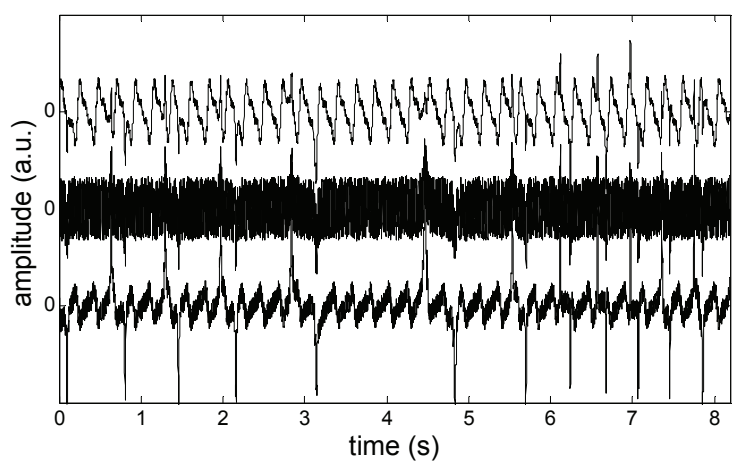

Figure 3: Mixture $\boldsymbol{z}$ after applying a unitary transform on the data of Figure 2.

In Table 2 and Table 3 we give the parameters of the data as displayed in Figure 2, respectively the results of the 
algorithm on the synthetic dataset. The results are given as the mean and standard deviation of the parameter with respect to the ground truth, i.e. the parameter defined on $x_{1}$ (respectively on the source with maximal spectral concentration in the $4-9 \mathrm{~Hz}$ band when the result is taken from an unordered ICA algorithm) with respect to the parameter of $z_{1}$ in the dataset.

\begin{tabular}{|c|ccc|}
\hline$\kappa$ & $\kappa^{c}$ & $f_{m}[\mathrm{~Hz}]$ & $S C[\%]$ \\
\hline-0.94 & 1200 & 4.64 & 73.44 \\
\hline
\end{tabular}

Table 2: Parameter values for the dataset given in Figure 2

\begin{tabular}{|r|llll|}
\hline contrast & \multicolumn{1}{c}{$\kappa$} & \multicolumn{1}{c}{$\kappa^{c}$} & $f_{m}[\mathrm{~Hz}]$ & $S C[\%]$ \\
\hline $\operatorname{Com} 2\left(\boldsymbol{x}^{*}\right)$ & 4.32 & -574.68 & -0.13 & -40.55 \\
{$[6]$} & \pm 7.71 & \pm 641.09 & \pm 2.21 & \pm 27.99 \\
\hline $\boldsymbol{\Psi}_{1}(\boldsymbol{x})$ & 0.21 & -768.33 & 0.04 & -34.30 \\
& \pm 0.22 & \pm 613.70 & \pm 0.56 & \pm 27.26 \\
\hline $\boldsymbol{\Psi}_{2}(\widetilde{\boldsymbol{x}})$ & 0.01 & -0.41 & 0.00 & -0.14 \\
& \pm 0.03 & \pm 7.12 & \pm 0.02 & \pm 0.33 \\
\hline
\end{tabular}

Table 3: Mean and Standard Deviation $(\mu \pm \sigma)$ for the error on the parameters over the 100 Monte Carlo realisations.

The above results show the (in)capability of an algorithm to find a signal $x_{1}$ (or $x_{i}$ in the case of com2) with similar parameters as $z_{1}$. Although the parameters determine the signal to a large extent, we give the Pearson's correlation coefficient (mean and standard deviation) with respect to each contrast: $\quad \rho_{\text {com } 2}=0.64 \pm 0.40, \quad \rho_{\Psi_{1}}=0.59 \pm 0.32$ and $\rho_{\Psi_{2}}=1.00 \pm 0.00$. For the critical reader, we express the latter in a higher precision to prevent any suspicion $\left(\rho_{\boldsymbol{\Psi}_{2}}=\right.$ $0.9977 \pm 0.0038)$.

Results on the real dataset in $\mathrm{F}$ are given in $\mathrm{F}$ and below. The algorithm is based on the $\boldsymbol{\Psi}_{2}(\omega)$ contrast defined on the interval $\Omega=[39] / f_{s}$, although similar results were obtained using the complete spectrum.

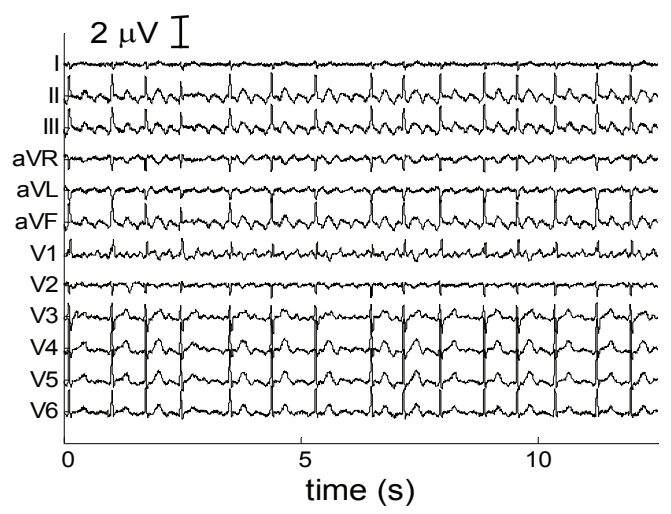

Figure 4: Patient ECG

The spectral concentration of the source estimate is $79.91 \%$ around the frequency of $3.58 \mathrm{~Hz}$. The kurtosis in the time and frequency domain are respectively -0.70 and 2145.00 .

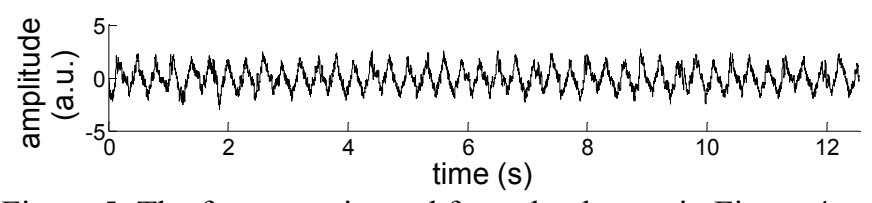

Figure 5: The f-wave estimated from the dataset in Figure 4

\section{Discussion}

Table 1 and Table 2 clearly support the choice of transforming the signals into the dual space of Fourier coefficients to estimate the source contribution from a complete set of observations. The results on the synthetic dataset leave no doubt about the method for the problem at hand. Moreover, real datasets tend to behave well under the assumptions made in the paper.

\section{Conclusion}

As can be readily derived from the results in Table 3 and the Pearson's correlation coefficients, transforming a signal in the appropriate domain and choosing the correct objective function leads to more accurate signal estimates in BSS. The synthetic data support the idea that the Fourier coefficients are an excellent candidate transform domain for f-wave extraction of the ECG.

\section{Acknowledgements}

The author acknowledges the FWO (Research Foundation Flanders) for the funding under contract number G.0095.05 and the Electrophysiology Laboratory of the University Clinical Hospital of Valencia, Spain and F. Castells for sharing the data with us.

\section{References}

[1] ACC/AHA/Physician Consortium 2008. "Clinical Performance Measures for Adults with Nonvalvular Atrial Fibrillation or Atrial Flutter", Circulation, pp. 1100-1120, (2008).

[2] G. Lee. "A Review of the Literature on Atrial Fibrillation: Rate Reversion or Control", Journal of Clinical Nursing, pp. 77-83, (2006).

[3] F. Castells, J.J. Rieta, J. Millet and V. Zarzoso. "Spatiotemporal Blind Source Separation Approach to Atrial Activity Estimation in Atrial Tachyarrhythmias", IEEE Trans on Biom Eng, pp 258-267, 52(2), (2005).

[4] V. Zarzoso, R. Phlypo and P. Comon. "A contrast for Independent Component Analysis with Priors on the Source Kurtosis Signs", IEEE Sig Proc Letters, accepted for publication.

[5] V. Zarzoso, A.K. Nandi, F. Herrmann and J. Millet. "Combined Estimation Scheme for Blind Source Separation with Arbitrary Source PDFs", IEE Electronics Letters, pp 132-133, 37(2), (2001).

[6] P. Comon. "Independent Component Analysis. A New Concept?", Signal Processing, pp 287-314, 36, (1994). 